Nutrition

Edited by Martin Wiseman MB FRCP FRCPath, Visiting Professor of Human

Nutrition and Alan Jackson MD FRCP, Professor of Human Nutrition

Institute of Human Nutrition, University of Southampton

\section{Nutrition in health}

\section{and disease}

Martin Wiseman MB FRCP FRCPath, Visiting

Professor of Nutrition

Alan A Jackson MD FRCP, Professor of Human Nutrition

Institute of Human Nutrition, Southampton General Hospital, Southampton

Clin Med 2004;4:397-400

Nutrition touches on every aspect of healthcare and medical practice. It may sometimes appear peripheral or coincidental, but often it is fundamental to a successful outcome. The underlying principles of nutrition and aspects of nutritional care should be integral to the training of every doctor. This article will consider selected areas of practice where common problems in nutrition are important, in order to illustrate the potential for effective nutrition in the promotion of health and disease and in the prevention and treatment of disease.

\section{Health promotion}

Health is achieved through an ongoing interaction with the environment to access the fundamental necessities for life, wholesome food, fresh air and clean water and is more than simply the absence of disease. During childhood it can be marked by normal growth and development, and during adulthood by the ability and opportunity to derive pleasure from engaging in socially valued activities. Consuming food enables the body's needs for energy and nutrients to be met. These needs vary with age and lifestyle, so there is variation in the amount and pattern of food which have to be consumed. Considerable processing, or metabolic exchange, is required within the body to modify food as consumed to a pattern of nutrients which meets the needs of individual cells and tissues of the body. The nature and extent of this processing vary amongst individuals depending on factors including genotype, past nutritional experience, lifestyle and current exposure to a range of environmental stressors. The capacity for the regulation and control of the metabolic exchange depends upon previous dietary exposure, body composition and nutrient status, especially micronutrient status.

Effective interventions to enable health by improving nutritional status are often indirect and not specifically aimed at improving food availability or diet. For example, improvements in personal hygiene, general sanitation, immunisa-

\section{Key Points} and treatment of many common diseases stresses disease routine practice requirement, nutritional state tion coverage or therapies for specific diseases (eg bed nets for malaria) can have major impact by reducing infective load, thereby ensuring that available nutrients can be best used for enabling normal development and function. Physical activity is an important attribute of health. Activity clearly confers cardiovascular benefit but also, by increasing energy expenditure, leads to increased food intake and hence increased nutrient intake if wholesome food is consumed. Indeed, activity can enable a diet of marginal nutrient density to become adequate because of increased consumption. Activity enables normal metabolic regulation and facilitates the appropriate partitioning and utilisation of nutrients within the body.

\section{Disease prevention}

Poor nutritional status is common and in its more extreme forms readily recognised as clinical disease. Less severe forms give rise to poor social functioning, low grade ill health and increased risk of more severe disease. During childhood poor health is seen frequently as a failure to develop adequately, readily identified as stunting, impaired neurocognitive development or impaired immune function. ${ }^{1}$ Extreme cases represent the most common cause of death: half of all deaths in children under the age of five years worldwide are associated with poor nutrition (about 10 million deaths each year).

Lesser degrees of impaired function

Nutrition is a fundamental determinant of health and important in the prevention

Lifetime nutritional exposure from conception to old age acts on genetic background to determine the body's capacity to cope with biological and other

Inappropriate nutrition during growth can have lasting impact on susceptibility to

Physicians need to include nutritional assessment and management as part of

KEY WORDS: chronic disease, environmental stressors, food intake, nutrient 
are carried into adult life. Barker ${ }^{2}$ and others have clearly demonstrated that poorer growth during early life is a major determinant of risk of chronic disease at later ages. Although most evident in the developing world, poor nutrition is seen in those groups in all societies who experience deprivation, poverty and the associated poor diet. As many as one in four poor urban households in the UK may experience hunger or food insecurity leading to ill health. ${ }^{3}$ Diets of poor quality and limiting in amount are underlying and fundamental causes of the major diseases in our society: heart disease, type 2 diabetes, cancer, obesity, mental ill health and a range of social problems (see McNeill and Cummings, and Treasure in this section). Stressful situations lead to increased nutrient losses which are difficult to correct if the background diet continues to be of marginal quality. The stresses may be imposed by biological factors such as infection, social or behavioural considerations such as smoking or psychological.

\section{Management and treatment of disease}

Patterns of disease vary in different countries $^{4}$ but the major causes of death in the UK are cardiovascular disease and cancer. In 2000 these were responsible for around $40 \%$ and $25 \%$ of deaths, respectively, in both men and women. Even within Europe there is wide variation in the risk of both of these conditions. ${ }^{4}$ For more than 50 years it has been clear that a major determinant of cardiovascular risk relates to nutritional factors, at least partly through established risk factors of blood concentrations of low-density lipoprotein cholesterol and blood pressure, and possibly also through other factors such as fruit and vegetable consumption. ${ }^{5}$ Migrant and other studies have implicated diet and nutrition as a principal factor in determining patterns of cancer. ${ }^{6,7}$

Nutrition is therefore a prime cause contributing to the commonest diseases leading to death and disability in the UK. An improvement in nutritional status based upon changes in the diet and patterns of food consumption and suitable levels of physical activity can help prevent a substantial part of this disease burden.

Better dietary patterns and increased physical activity are effective components of the secondary treatment of patients with established heart disease, type 2 diabetes, obesity, cancer and mental ill health. At the same time, the presence of disease influences nutrition status, through its effects both on food and nutrient intake, on the altered nutrient needs of the body and as a result of increased nutrient losses during illness. There is considerable evidence that nutrition status has an important influence on clinical course in acute illness $^{8}$ (see Elia and Stroud in this section).

\section{Nutritional status}

\section{Measurement}

Measuring nutritional status reliably is a skilled task, but reasonable indications can be obtained based on a historical assessment of:

- dietary intake

- anthropometric indicators of body size and composition (height, weight, waist circumference), and

- markers of metabolic capability or the function of individual tissues (biochemical markers, grip strength, work capacity).

Body composition and functional measures indicate the extent to which the diet has been adequate to meet the needs of the body. In the short term, any inability to meet the needs of the tissues may be buffered by drawing on functional reserves in tissues such as muscle, adipose tissue and bone, leading to changes in body composition. With time, there are direct consequences for tissue function, leading to evident disease (see Gariballa in this section).

\section{Appetite}

A major determinant of dietary intake is appetite, which is only imperfectly responsive to the needs of the body. Mechanistically, appetite is determined by a complex interaction of peripheral and central hypothalamic feedback systems including a response to activity. In health, the hunger response to reduced food intake is highly conserved, while suppression of hunger as a result of feeding is less strong, especially in people who are inactive, thus increasing the likelihood of overweight and obesity. Eating behaviour is also influenced by psychological factors. Food choice is influenced by personal attitudes and beliefs and, to a lesser extent, knowledge. Perhaps more important is the influence of conformation to perceived norms, such as peer group pressure or cultural practice. Social factors such as income or food availability are important determinants of choice or lack of choice. ${ }^{9}$

\section{Responding to environmental challenge}

The nutrient environment of each cell plays an important determinant role in the pattern and extent of gene expression. The requirement of the body for energy and nutrients is determined by size and activity, by physiological changes such as pregnancy, and the pathological changes of disease. For each individual there is a cumulative nutritional experience throughout life against the background of individual genotype. There are particular stages of development during which vulnerability to nutritional limitation is greatest and where relatively modest adverse experience carries a longer lasting effect, the ultimate outcome being determined by the timing, intensity and duration of the adverse experience. Life presents ongoing environmental challenges. The capability of an individual to withstand or cope with differing degrees of social, psychological or biological stress is determined by the nature of the cumulative experience to nutritional exposures against the background of an individual's genotype.

In responding to environmental challenge with a stress, inflammatory or immune response there is a delicate balance between an adequate and appropriate response and either an inadequate or an excessive response. Poor nutritional status or poor food intake modulates the nature and intensity of 
these responses, both generally and specifically, leading to reduced resistance and increased susceptibility to such challenges. Nutrient effects can also lead to an excessive response. For example, the pattern of fatty acids can lead to an imbalance between pro- and anti-inflammatory processes which is sensitive to individual genotype, resulting in clinical disease such as asthma, rheumatoid arthritis, heart disease or metabolic syndrome (see Gariballa in this section).

Fig 1. Cumulative nutritional/metabolic experience. Maintenance of health and prevention of disease results from a lifelong interaction between genetic background, early life experience with programmed susceptibility and current exposures. III health can be manifest as a deviation from normal either in the direction of over- or undernutrition. Health promotion encompasses, but goes beyond, disease prevention and forms part of a spectrum of management of disease associated with various nutritional states.

\begin{tabular}{|c|}
\hline $\begin{array}{c}\text { Gene } \\
\text { mother/father }\end{array}$ \\
\hline $\begin{array}{c}\text { Embryo } \\
\text { preconception } \\
\text { Fetal } \\
\text { pregnancy }\end{array}$ \\
\hline Infancy \\
\hline Childhood \\
\hline Adolescence \\
\hline Adulthood \\
\hline
\end{tabular}

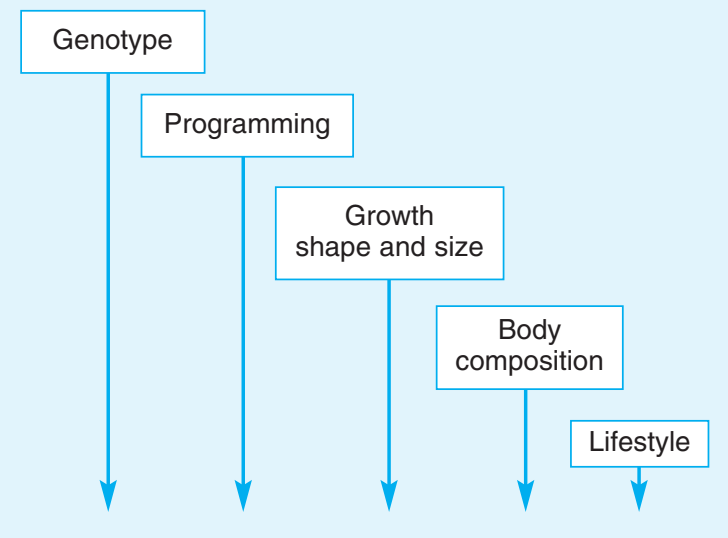

HEALTH AND WELL-BEING or DISEASE

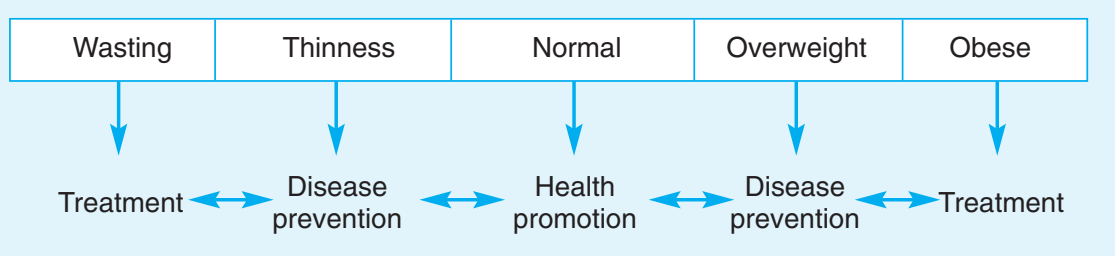

and those recommended for promoting health. Physicians occupy an important and influential position in addressing these failures, both directly in regard to their own patients and indirectly through colleagues and other health professionals.

\section{References}

1 Bhutta ZA. Beyond Bellagio: addressing the challenge of sustainable child health in developing countries. Review. Arch Dis Child 2004;89:483-7.

2 Barker DJ. Fetal programming of coronary heart disease. Review. Trends Endocrinol Metab 2002;13:364-8.

3 Acheson D. Inequalities in Health: an Independent Inquiry. London: The Stationery Office, 1998.

4 World Health Organisation. Diet, Nutrition and the Prevention of Chronic Diseases. Technical Report Series no 916. Geneva: WHO, 2003.

5 COMA. Nutritional Aspects of Cardiovascular Disease. London: The Stationery Office, 1994.

6 COMA. Nutritional Aspects of the Development of Cancer. London: The Stationery Office, 1998.

7 World Cancer Research Fund. Food, Nutrition and the Prevention of Cancer: a Global Perspective. Washington: WCRF/American Institute for Cancer Research, 1997.

8 World Health Organization. Management of severe malnutrition: a manual for physicians and other senior health workers. Geneva: WHO, 1998.

9 Lobstein T, Baur L, Uauy R; IASO International Obesity Task Force. Obesity in children and young people: a crisis in public health. Review. Obes Rev 2004;5(Suppl 1): $4-104$.

10 Wanless D. Securing our Future Health: Taking a Long Term View. London: The Stationery Office, 2002.

11 Department of Health. Choosing Health: a consultation on action to improve people's health. London: DH, 2004.

\section{Fig 2. Cumulative}

nutritional/metabolic

experience: capacity to cope.

Nutritional state can be

characterised as the ability of the

body to maintain function in the

face of external environmental

stressors which may be biological, psychological or social.

Responses to these stressors may manifest in a number of ways; when the body's capacity to cope is exceeded, decompensation leads to disease.

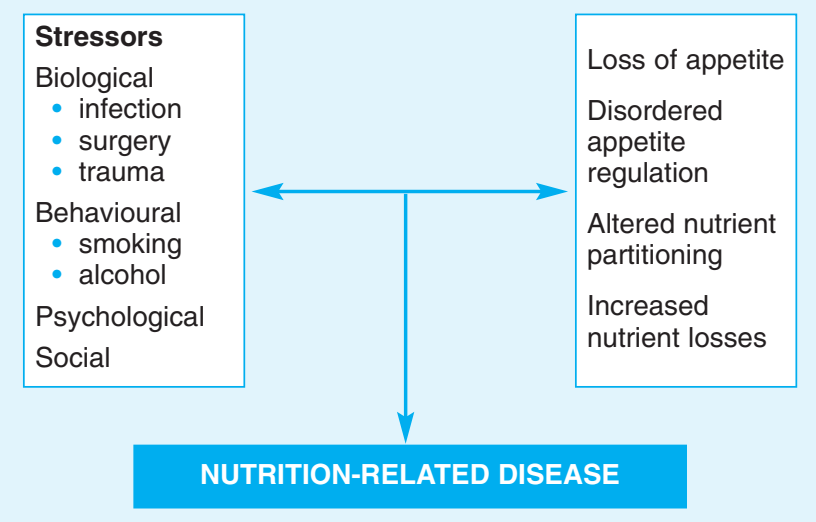


12 Royal College of Physicians. Nutrition and patients: a doctor's responsibility. Report of a working group. London: RCP, 2002.

13 Lennard-Jones J E (ed). A Positive Approach to Nutrition as Treatment. London: King's Fund Centre, 1992.

14 McWhirter JP, Pennington CR. Incidence and recognition of malnutrition in hospital. BMJ 1994;308:945-8.
Nutrition and

common health

\section{problems}

Geraldine McNeill MB ChB Phd, Research Fellow in Epidemiology, Department of Environmental and Occupational Medicine, University of Aberdeen, Foresterhill, Aberdeen

John H Cummings FRCP, Professor of Experimental Gastroenterology, Division of Pathology and Neuroscience, University of Dundee, Ninewells Hospital and Medical School, Dundee

\section{Clin Med 2004:4:400-4}

In the past fifty years, the UK diet has changed substantially ${ }^{1}$ while physical activity has decreased, resulting in a rapid increase in overweight and obesity. These changes have coincided with increases in the incidence of many chronic diseases such as non-insulin dependent diabetes mellitus (NIDDM), some cancers, osteoporosis and bowel disease. Many dietary components have a role in both prevention and management of these diseases (Table 1). Evidence for the role of diet and physical activity in the aetiology of chronic disease relies principally on the consistency of epidemiological data supported by evidence of mechanisms and some intervention studies. The World Health Organization recently summarised the evidence for relationships between diet and physical activity and the development of chronic diseases (Table 2). ${ }^{2}$

\section{Cardiovascular disease}

\section{Fats}

High serum concentrations of cholesterol, particularly low-density lipoprotein (LDL) cholesterol and low levels of high-density lipoprotein (HDL) cholesterol, predict coronary heart disease mortality. The use of statins to lower LDL
Table 1. Food sources of nutrients associated with chronic disease risk in the UK diet.

- Nutrients associated with increased risk of chronic disease

\begin{tabular}{ll}
\hline Nutrient & Food sources \\
\hline Fat & Fried foods, pastries, cakes, crisps, rich sauces \\
Saturated fatty acids & High fat dairy foods, processed meats \\
Trans fatty acids & Biscuits, cakes, pastries, hard margarines \\
Sugar & Sweetened drinks, confectionery, biscuits \\
Sodium & Processed foods, savoury snacks \\
& \\
- Nutrients associated with decreased risk of chronic disease \\
\hline Nutrient & Food sources \\
\hline Mono-unsaturated fatty acids & Olive oil, rape seed oil, nuts, avocados \\
n-6 polyunsaturated fatty acids & Sunflower and corn oils, margarines \\
n-3 polyunsaturated fatty acids & Oily fish, rape seed oil, leafy vegetables \\
Fibre & Wholegrain cereals, vegetables, beans, pulses \\
Vitamin C & Citrus fruits and juices, potatoes \\
Folic acid & Fortified cereals, leafy vegetables, orange juice, liver \\
Vitamin E & Wholegrain cereals, fortified margarines, vegetable oils \\
Vitamin D & Fish oils, fortified margarines, liver \\
Calcium & Milk, cheese, yogurt, wholegrain cereals, soya \\
Potassium & Fruits, vegetables \\
\hline 'Makel, &
\end{tabular}

"Mackerel, herring, salmon, sardines, pilchards, trout and fresh (but not canned) tuna. 